\title{
A Novel Quantitative, Multianalyte Immunoassay to Detect Neuroinflammation following Traumatic Brain Injury
}

R\&D Systems, Inc., 614 McKinley Place NE, Minneapolis, MN 55413

ProteinSimple, 3001 Orchard Parkway, San Jose, CA 95134

\section{Introduction}

Traumatic brain injury (TBI) affects millions of people worldwide every year. To help diagnosis TBI and monitor the recovery process, researchers have been attempting to identify molecular changes that occur in the brain. Following brain injury, proinflammatory biomarkers are released and induce a neuroinflammatory response. The prolonged presence of these biomarkers can affect neurons and brain functioning. Inflammation can also alter cell phenotypes and cause increased cytokine production and neural damage. Unfortunately, a single blood inflammatory biomarker may not indicate brain pathology. Evaluating multiple
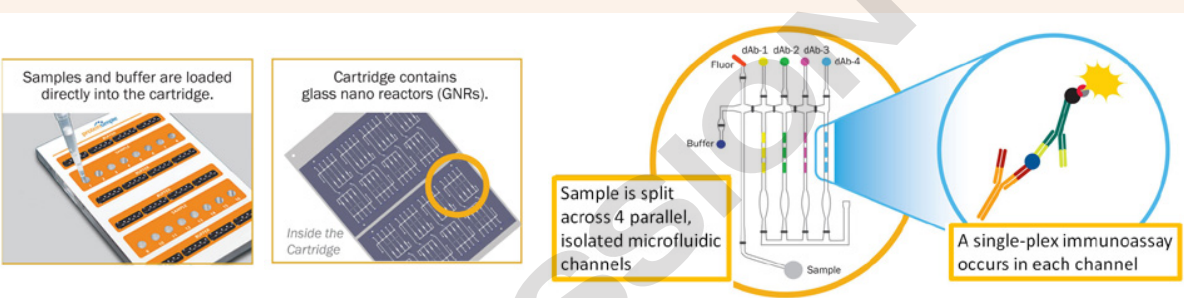

Figure 1. Simple Plex ${ }^{\mathrm{TM}}$ is a fully automated, quantitative multianalyte immunoassay. Setup only takes 5 minutes. Samples are simply pipetted into the cartridge, which automates the entire assay. Data is ready in 60 minutes. biomarkers may be more informative and allow for a more accurate diagnosis. However, many times, sample volume limits the number of analytes that can be assayed. Simple Plex ${ }^{\mathrm{TM}}$, a novel quantitative, multianalyte immunoassay, overcomes this problem. Simple Plex ${ }^{\mathrm{TM}}$ is a parallel multianalyte detection technique that delivers high precision and accuracy with $\leq 25 \mu \mathrm{L}$ of sample. We used this immunoassay platform to evaluate 14 neuroinflammatory markers in serum and plasma samples from individuals diagnosed with TBI.

\section{Materials and Methods}

Serum and plasma samples from individuals diagnosed with varying symptoms of TBI were purchased from Discovery Life Sciences. The levels of 14 analytes, BAFF/BLyS/TNFSF13B, CCL2/MCP-1, CHI3L1/YKL-40, CXCL8/IL-8, CXCL10/IP-10, EGF, HGF, IL-1//LL-1F2, IL-6, IL-10, IL-15, Proprotein Convertase 9/PCSK9, TNF- $\alpha$, and VEGF, were analyzed in these samples (N=15) and in serum and plasma samples from apparently healthy individuals $(\mathrm{N}=15)$ using the Simple Plex ${ }^{\mathrm{TM}}$ multianalyte immunoassay platform (ProteinSimple). TBI and control samples were diluted either 1:2 or 1:10 with Sample Diluent and vortexed prior to assaying.

\section{Results and Discussion}

- The concentrations of nine analytes were higher in TBI samples compared to control samples: CCL2/MCP-1, CHI3L1, CXCL8/ IL-8, CXCL10/IP-10, EGF, HGF, IL-6, IL-10, and IL-15.

- The concentrations of five analytes were not different between TBI and control samples: BAFF/BLyS/TNFSF13B, IL-1//L-1F2, Proprotein Convertase 9/PCSK9, TNF- $\alpha$, and VEGF.

\section{Conclusions}

We compared the levels of proteins in serum samples from control individuals and individuals diagnosed with TBI using the Simple Plex ${ }^{\top \mathrm{M}}$ platform. We found that the concentrations of several proteins that are used as markers of inflammation and neural damage were higher in serum from TBI patients compared to control serum. Our results also demonstrate the broad utility of the Simple Plex ${ }^{\mathrm{TM}}$ platform as a tool for discovery by allowing the comparison of multiple biomarkers simultaneously from a single small sample volume with very high sensitivity.

To learn more, please visit: rndsystems.com/Immunoassays.

BioTechniques 60:153 (March 2016) doi 10.2144/000114395 